\title{
Limitations in transplantation of astroglia-biomatrix bridges to stimulate corticospinal axon regrowth across large spinal lesion gaps
}

\author{
Ronald Deumens $^{\mathrm{a}, \mathrm{c}}$, Guido C. Koopmans ${ }^{\mathrm{a}, \mathrm{b}, \mathrm{c}}$, Wiel M.M. Honig ${ }^{\mathrm{a}, \mathrm{b}}$, Véronique Maquet $^{\mathrm{d}}$, Robert Jérôme ${ }^{\mathrm{d}}$, Harry \\ W.M. Steinbusch ${ }^{\mathrm{a}, \mathrm{c}}$, Elbert A.J. Joosten ${ }^{\mathrm{b}, \mathrm{c}}$ \\ ${ }^{a}$ Department of Psychiatry and Neuropsychology, Division Cellular Neuroscience, University of Maastricht, \\ P.O. Box 616, 6200 MD, Maastricht, The Netherlands \\ ${ }^{\mathrm{b}}$ Department of Anesthesiology, Academic Hospital Maastricht, P. O. Box 5800, 6202 AZ, Maastricht, The \\ Netherlands \\ ${ }^{\mathrm{c}}$ European Graduate School of Neuroscience (EURON), University of Maastricht, P.O. Box 616, 6200 MD, \\ Maastricht, The Netherlands \\ ${ }^{\mathrm{d}}$ Center for Education and Research on Macromolecules, University of Liège, Sart-Tilman B6, 4000 Liège, \\ Belgium
}

\begin{abstract}
Regrowth of injured axons across rather small spinal cord lesion gaps and subsequent functional recovery has been obtained after many interventions. Long-distance regeneration of injured axons across clinically relevant large spinal lesion gaps is relatively unexplored. Here, we aimed at stimulating long-distance regrowth of the injured corticospinal (CS) tract. During development, an oriented framework of immature astrocytes is important for correct CS axon outgrowth. Furthermore, a continuous growth promoting substrate may be needed to maintain a CS axon regrowth response across relatively large spinal lesion gaps. Hence, we acutely transplanted poly(D,L)-lactide matrices, which after seeded with immature astrocytes render aligned astrocyte-biomatrix complexes (R. Deumens, et al. Alignment of glial cells stimulates directional neurite growth of CNS neurons in vitro. Neuroscience 125 (3) (2004) 591-604), into 2-mm long dorsal hemisection lesion gaps. In order to create a growth promoting continuum, astrocyte suspensions were also injected rostral and caudal to the lesion gap. During 2 months, locomotion was continuously monitored. Histological analysis showed that astrocytes injected into host spinal tissue survived, but did not migrate. None of the astrocytes on the biomatrices survived within the lesion gap. BDA-labeled CS axons did not penetrate the graft. However, directly rostral to the lesion gap, $120.9 \pm 38.5 \%$ of the BDA-labeled CS axons were present in contrast to $12.8 \pm 3.9 \%$ in untreated control animals. The observed anatomical changes were not accompanied by locomotor improvements as analyzed with the BBB and CatWalk. We conclude that although multifactorial strategies may be needed to stimulate longdistance CS axon regrowth, future studies should focus on enhancing the viability of cell/biomatrix complexes within large spinal lesion gaps.
\end{abstract}

Keywords: Rat; Spinal cord injury; Polylactides; Alignment; Corticospinal; Locomotion

Functional recovery after injury to the central nervous system (CNS) is only very limited. The main cause is the absence of spontaneous axon regeneration or regrowth due to an unpermissive environment of the injured CNS. Axon regrowth and partial functional recovery after spinal cord injury (SCI) can be stimulated by manipulating the injured spinal cord environment. The longest descending axon tract in the spinal cord, the corticospinal (CS) tract, has been elicited to regrow after injury upon transplantation of growth promoting cells. Various cellular transplants have been used [5], including among others genetically modified cells [8] and olfactory ensheathing cells $[13,14,18]$. However, the SCI models that were used involve single-cut lesions and electrolytic lesions, with which relatively small spinal lesion gaps are associated. The lesion gaps in the injured human spinal cord can be relatively long. Long-distance regeneration of injured CS axons across relatively large spinal lesion gaps (i.e. several millimeters in a rostrocaudal plane) has, thus far, been unsuccessful after transplantation of growth promoting cells, such as olfactory ensheathing cells [3] or immature astrocytes [11]. Although injured CS axons did not display long-distance regeneration, they were found to regrow into or underneath the cellular grafts that were transplanted into the relatively large lesion gaps. The lack of long-distance CS axon regeneration may have been influenced by two issues. First, the transplanted glia may have lacked a clear orientation. Developmental studies have shown that an oriented astroglial framework is crucial for the correct long-distance outgrowth of the CS tract [10]. Also, aligned complexes of astrocytes on biologically degradable matrices have been shown to 
stimulate directional regrowth of CNS neurons, including CS neurons [4]. Second, no continuum of transplanted glia was formed in the injured spinal cord [11], which may have restricted the regrowing CS axons to the area of the transplanted glia.

In the present study we developed a multifactorial strategy that combines orientation of the main cells responsible for CS axon growth during development (i.e. immature astrocytes) with a growth supporting (i.e. astroglial) continuum in the injured spinal cord. This strategy consisted of (1) acute transplantation of aligned astrocyte-biomatrix complexes [4] into a 2-mm long dorsal hemisection lesion gap, and (2) acute injections of immature astrocytes rostral and caudal to the lesion gap. Obviously, migration of these injected immature astrocytes from the injection sites towards the spinal lesion site is a prerequisite for the creation of an astrocyte continuum in the injured spinal cord.

Astrocytes were obtained from neonatal (post natal day 1) Lewis rats. The method of astrocyte culturing has been described elsewhere $[4,6]$. Fourteen days old cultures containing $>95 \%$ GFAP positive astrocytes were plated onto the surface area of PLA/PLA- $b$-PEO matrices [16] at a density of about $40,000 \mathrm{cells} / \mathrm{cm}^{2}$ and grown on these substrates for 4 days. This protocol rendered aligned astrocyte-biomatrix complexes [4] and these complexes were used for transplantation into the spinal lesion site. Identical astrocyte cultures, obtained from the same source (Lewis rat pups) were maintained in Petridishes till the day of transplantation and used for the cell injections into the rostral and caudal spinal cord stumps. One day before transplantation, both the astrocytebiomatrix complexes and the astrocyte cultures were prelabeled with Hoechst. This was done by a 40-60 min incubation at $37{ }^{\circ} \mathrm{C}$ in $2 \mu \mathrm{g} / \mathrm{ml}$ Hoechst 33342 (Sigma, Uithoorn, The Netherlands) in culture medium: DMEM/NUT mix F12 with glutamax-I (Gibco/Invitrogen, Breda, The Netherlands), supplemented with 10\% inactivated fetal calf serum (Bodinco, Alkmaar, The Netherlands) and antibiotics (100 U/ml penicillin and 100 $\mu \mathrm{g} / \mathrm{ml}$ streptomycin). Thereafter, the astrocyte-biomatrix complexes and the astrocyte cultures were extensively washed with culture medium.

Twenty-seven male Lewis rats (inbred, animal facilities of Maastricht University), 7 weeks old, 200-250 g in body weight, were pretrained for CatWalk behavioral testing as described previously [12]. When the animals were able to make three consecutive runs on the CatWalk without hesitation, preoperative CatWalk data were obtained. Next, the animals received Temgesic $(0.1 \mathrm{mg} / \mathrm{kg}$ body weight; subcutaneous injections; ScheringPlough, Utrecht, The Netherlands) one hour before surgery. Anesthetization was performed using a mixture of $\mathrm{O}_{2} / \mathrm{N}_{2} \mathrm{O}(1: 2)$ and halothane (5\% for induction of anesthesia and 1-2\% as a maintenance dose). A laminectomy was performed at T11/T12 and the dura was incised. Using microscissors the dorsal half of the spinal cord was incised at two spinal levels, $2 \mathrm{~mm}$ apart. The dorsal part of the spinal cord in between the two cuts was removed with an aspiration device. The experimental group $(n=12)$ received astrocyte-biomatrix complexes into the lesion site and two astrocyte suspension injections $(100,000$ cells $/ \mu 1 ; 2 \mu l$ per injection site; 1 injection site/stump), one at $1 \mathrm{~mm}$ rostral and the other at $1 \mathrm{~mm}$ caudal to the lesion site, both at a depth of $1.2 \mathrm{~mm}$. Dura Film was used to cover the lesion site. Control animals $(n=15)$ did not receive a transplant into the lesion site, but received culture medium injections into the rostral and caudal stumps to control for possible damage induced by the injection procedure. The wounds were sutured and the animals returned to their home cages. At 6 weeks after injury, BDA labeling of the CS tract was performed in the animals via an identical method as described previously [11].

At 9 weeks after SCI, i.e. 3 weeks after CS tract tracing, all animals were anesthetized (Nembutal, $30 \mathrm{mg} / \mathrm{kg}$ body weight; i.p. injection) and transcardially perfused with ice-cold $4 \%$ paraformaldehyde in $0.1 \mathrm{M}$ phosphate buffer ( $\mathrm{pH}$ 7.4). The spinal cords were removed and post-fixed in cold $4 \%$ buffered paraformaldehyde overnight. The cryoprotection protocol consisted of an overnight incubation in $10 \%$ sucrose in PBS $\left(0.1 \mathrm{M} ; \mathrm{pH} 7.6 ; 4^{\circ} \mathrm{C}\right)$ followed by a 3-day incubation in $25 \%$ sucrose in PBS at $4^{\circ} \mathrm{C}$. Thereafter, $2.5-\mathrm{cm}$ long spinal cord pieces including the lesion site were frozen and stored at $-80^{\circ} \mathrm{C}$. The spinal cord pieces were serially cut on a cryostat (sagittal sections of $25 \mu \mathrm{m}$ ) and the sections were immediately mounted on gelatin chrome-alumn (Sigma, Uithoorn, The Netherlands) coated glass slides and stored at $-20^{\circ} \mathrm{C}$. Alternate sections were processed for immunohistochemistry. Sections were washed in the following sequence: $0.3 \%$ Triton X-100 in Tris-buffered saline (TBS-T), TBS, and TBS-T (10 min each step). Rabbit anti-GFAP (1:1000; DAKO, Glostrup, Denmark) and rabbit anti-GAP43/B50 (generous gift from Leo van Halewijn, Utrecht University, The Netherlands) were used as primary antibodies, diluted in TBS-T and incubations were overnight at room temperature. After washing, the sections were incubated with secondary antibodies for $1.5 \mathrm{~h}$ at room temperature. The secondary antibodies used were streptavidin-Cy3 (1:2000; for the BDA-labeled CS axons; Jackson ImmunoResearch Europe Ltd., Cambridgeshire, UK) and Alexa488-conjugated goat anti-mouse (1:100; Molecular Probes/Invitrogen, Breda, The Netherlands). Stained sections were analyzed using an Olympus AX-70 microscope using epi-fluorescent illumination. The microscope was equipped with a $\mathrm{x} 20$ objective and a $\times 10$ 
projection lens. Pictures of the stained sections were made at a magnification of x20 using an Olympus F-view cooled CCD camera (Paes, Zoeterwoude, The Netherlands). All pictures were analysed with the image analyzing system analySIS Vers. 3.0. (Soft Imaging System, Munster, Germany).

All sections with BDA labeled CS axons were analyzed. For quantitative analysis, $250-\mu$ m-wide-boxes were placed at four different locations in the spinal cord sections (Fig. 1D). The first box was placed in the dorsal grey/white matter at $2.5-3 \mathrm{~mm}$ rostral to the injury site and will be designated as "the rostral reference". The second box was placed in the dorsal grey/white matter area directly rostral to the injury site. The third box was placed in the grey matter underneath the lesion site. Since only few axons were present underneath the lesion site, the box was placed in the area where most BDA immunore-activity was found. The fourth box was placed in the grey matter caudal to the injury site, again at the position where most BDA immunoreactivity could be found. The few BDA immunoreac-tive CS axons were exclusively present within the caudal grey matter within $10 \mathrm{~mm}$ from the caudal edge of the lesion site. All four boxes were analyzed in all the sections containing BDA labeled CS axons. The percentage of each box occupied by BDA immunoreactive axons ("BDA immunoreactivity occupation") was measured and the box values in all the sections were summed. Since the efficiency of CS tracing with BDA was highly variable among animals, the BDA immunoreactivity at the rostral reference was taken as the $100 \%$ reference value of BDA labeled axons of each animal. The BDA immunoreactivity values of boxes 2,3 , and 4 were expressed relative to that of the rostral reference. A similar quantification method has been described previously by others [7].

Behavioral evaluation of the animals was performed throughout the 9 weeks post-operative period, using the BBB locomotor rating scale [1] and the CatWalk gait analysis [9], the latter according to previous recommendations [12].

In saggital spinal cord sections of all astrocytes-transplanted animals, many Hoechst-prelabeled cells were detected around both the rostral and caudal injection sites. GFAP immunostain-ing confirmed that these Hoechst cells were indeed astrocytes (Fig. 1A). However, prelabeled astrocytes were not detected at or near the biomatrix complex that was located within the lesion site. BDA-labeled CS axons were clearly visible rostral to the injury site in all astrocyte-transplanted animals and in all-but-one control animal. Many retraction bulbs were observed at the endings of these BDA-labeled CS axons in both transplanted and control animals (Fig. 1B and C). The majority of BDA-labeled CS axons stopped at the rostral lesion border and only very few grew in the sublesional grey matter and in the caudal grey matter. No BDA-labeled CS axons were observed to penetrate the lesion/graft area. Quantitatively, a significantly enhanced BDA immunoreactivity was detected directly rostral to the injury site (box 2 in Fig. 1D) in astrocyte transplanted $(n=7)$ versus control animals $(n=7)(p<0.05)$ (Fig. 1E). None of the BDA-labeled CS axons in this study was found to be immunoreactive for GAP43/B-50, a marker molecule known to be involved in axon growth $[19,21]$.

Behaviorally, the animals $(n=15)$ showed a significant drop in BBB score directly after injury followed by a gradual increase to a plateau-phase (Fig. 1F) as reported earlier [11]. Although there was a clear time effect for the BBB score (Time: $F_{12,13}=170.48, p<0.01$ ), there was no difference between groups (Group: $F_{1,13}=0.77$, n.s.). Next to this, many CatWalk parameters were tested. Among these, the stride length of the hind limbs, which has been linked to the CS tract [20], was significantly reduced after injury (Time: $F_{1,13}=20.40, p<0.01$ ) with no difference between groups (Group: $F_{1,13}=0.058$, n.s.) (Fig. $\left.1 \mathrm{G}\right)$.

This study describes a multifactorial transplantation strategy which was unsuccessful in stimulating regrowth of injured CS axons across relatively large lesion gaps. This strategy only enhanced the presence of injured CS axons detected directly rostral to the lesion gap. This was not paralleled by any functional improvement till 9 weeks after injury, as assessed with the BBB locomotor rating scale or CatWalk gait analysis.

Among cell transplantation strategies to stimulate CS axon regrowth across discrete electrolyte or single-cut spinal lesion sites, olfactory ensheathing cell (OEC) transplants have been shown to be useful [13,18]. Clinically, relatively large spinal lesion gaps of at least several millimeters occur and the use of OEC transplants to stimulate CS axon regrowth across such larger lesions has been more limited [3]. Long-distance CS axon growth, however, occurs during development and a continuous oriented framework of immature astrocytes is thought to be responsible [10]. In a first study, immature astrocytes were embedded in a collagen matrix and subsequently transplanted into 2-mm long dorsal hemisection lesion gaps [11]. These transplanted astrocytes most likely did not adopt an oriented phenotype and were only present within the spinal lesion gap [11]. From previous work we know that the use of oriented $\rho$ oly $(\mathrm{D}, \mathrm{L})$-lactide matrices enables the creation of aligned astrocyte-biomatrix substrates [4]. Although the present study aimed at creating (1) an aligned astrocytebiomatrix substrate within the lesion gap, and (2) a continuum of immature astrocytes within the lesioned spinal 
cord, both of these aims were not achieved. Hoechst-prelabeled transplanted cells were only detected in a clustered fashion within the injection areas rostral and caudal to the lesion gap. No prelabeled cells were detected on the poly(D,L)-lactide matrices, which strongly suggests that these transplanted cells did not survive on these matrices within the lesion gaps. An alternative explanation for the lack of prelabeled cells on the biomatrices is that they migrated away from the biomatrices in the direction of the injection areas. However, since the prelabeled cells within these injection areas were strongly clustered and no putative migratory tracts could be observed this explanation is rather unlikely.

Fig. 1. Neuropathological and behavioral effects of astrocyte transplantation. Hoechst-prelabeled (blue) transplanted astrocytes, immunoreactive for GFAP (red) readily survived within the injection areas rostral (A) and caudal to the spinal lesion gap. Note in panel (A) that autofluorescent macrophages can be detected by yellowish fluorescence. BDA-labeled corticospinal axons approached the spinal lesion site more closely in astrocyte transplanted animals (B) than in control animals (C). Note that panels B and C are taken directly rostral to the lesion gap (box 2 in panel D) and in both panels retraction bulbs can be observed at the end of the BDAlabeled CS axons. (D) Four different boxes were placed in the injured spinal cord. BDA immunoreactivity was measured in these boxes; box 1 was taken as a reference box. Quantitative analysis showed a significantly reduced die-back of corticospinal axons in astrocyte transplanted animals versus control animals directly rostral to the injury site (E). Behavioral analysis using the BBB locomotor scale $(F)$ and the CatWalk gait analysis $(\mathrm{G})$ showed that the astrocyte intervention did not stimulate any recovery as compared to control animals. DPO, days post operation; $\mathrm{w}$, white matter; $\mathrm{g}$, grey matter; $\mathrm{t}$, transplant; BDA, biodextran amine; $* p<0.05$.
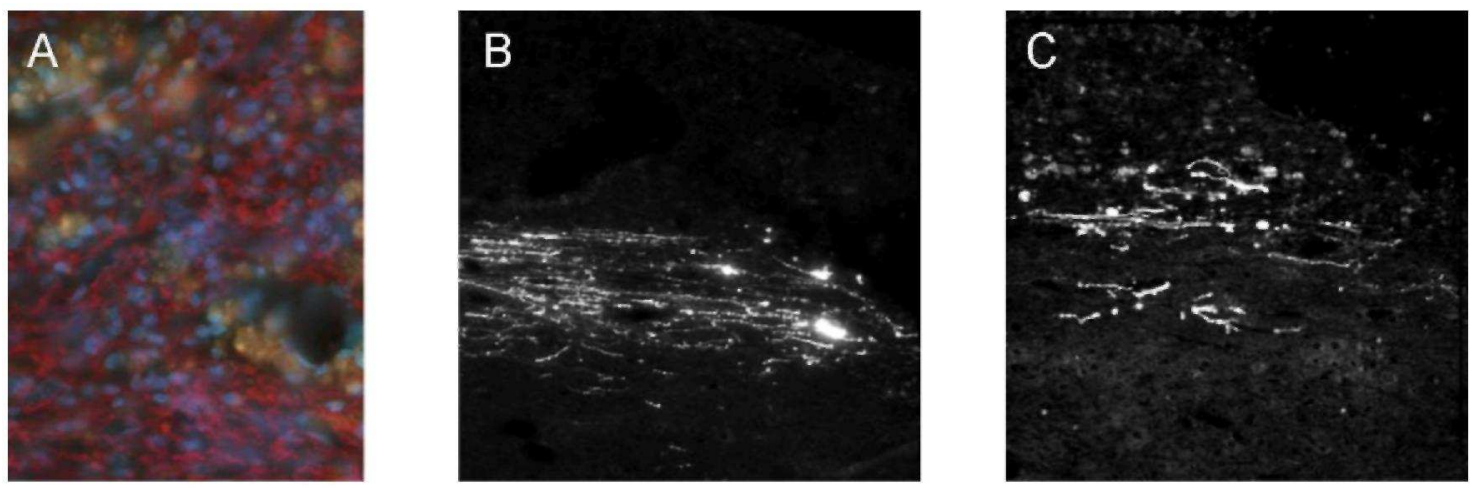

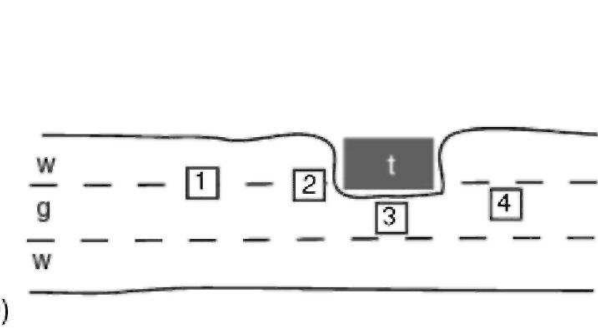

(D)

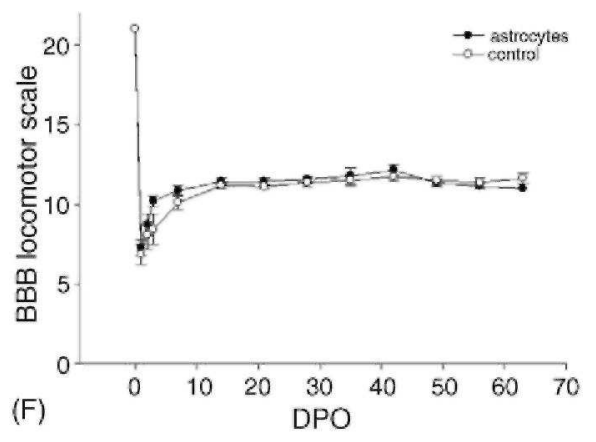

(E)
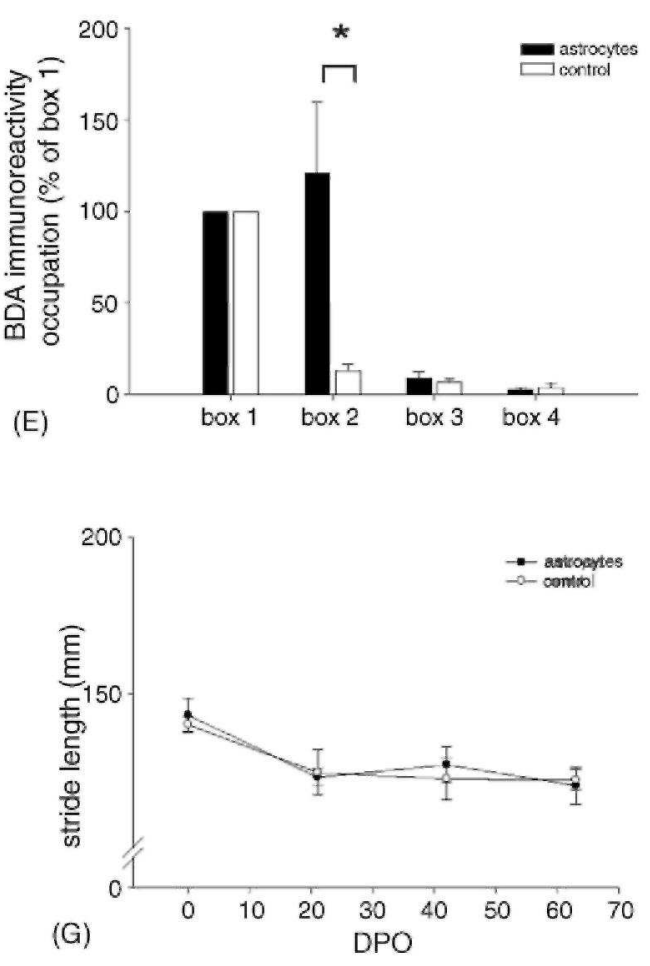
The survival of transplanted immature astrocytes on the poly $(\mathrm{D}, \mathrm{L})$-lactide matrices may have been negatively influenced by the rather 'unprotected' location. When immature astrocytes were embedded within a collagen matrix and then transplanted into 2-mm long dorsal hemisection sites, the transplanted astrocytes readily survived [11]. However, in the present study the astrocytes were cultured merely on the surface of the $\rho$ oly(D,L)lactide matrix, which renders these cells directly exposed to the high concentrations of cytotoxic substances released by pathological processes, such as hemorrhage and inflammation [17], which may have negatively affected the survival of these cells. In addition, it has been shown that injured spinal cord tissue influences the well-being of Schwann cells [22], and may also exert detrimental effects on the survival of astrocytes transplanted into our dorsal hemisection lesion gaps. Survival of transplanted immature astrocytes may benefit not only from culturing these cells within biomatrices, but also from the specific biomaterial used. Degradation of polylactides is associated with a lowering of the $\mathrm{pH}$ [15], which may also negatively affect the survival of the transplanted immature astrocytes. Hence, future studies may focus on the development of aligned astrocytebiomatrix complexes, which are more viable after transplantation in spinal lesion gaps.

Although our strategy was associated with no survival of the transplanted cells within the lesion gap and poor migration of the transplanted cells from the injection sites, BDA immunoreactivity directly rostral to the lesion gap equaled that at 2.5-3 mm rostral to the lesion gap. In contrast, control animals here showed an over $85 \%$ reduction in BDA immunoreactivity $(p<0.05)$. BDA-labeled CS axons were never observed to penetrate the biomatrices, which may reflect the rather low intrinsic capacity of injured CS axons to regrow [5]. Other studies also showed the inability of injured CS axons to use organic implants, such as human amnion ECM material, collagen implants and laminin-coated nitrocellulose filters to cross lesion gaps [2]. Of the rostrally located injured BDA-labeled CS axons, none was immunoreactive for GAP43/B50 in neither transplanted nor control animals. This suggests that the higher BDA immunoreactivity directly rostral to the lesion gap in transplanted animals reflects a die-back response of these axons, rather than a sprouting response. The nature of this die-back response may be investigated in future studies focusing on the BDA-labeled CS axons at subsequent time points after injury and how these axons are affected by the transplanted immature astrocytes. Electronmicroscopic analysis may be used to see whether the enhanced BDA immunoreactivity immediately rostral to the lesion gap after astrocyte transplantation involves a swelling of the BDA-labeled CS axons and/or a reduction in the number of BDA-labeled CS axons retracting. Interestingly, we never observed a contact between the transplanted astrocytes and the BDA-labeled CS axons. Hence, the effects of the rostrally injected astrocytes on the injured CS axons are most probably mediated by diffusible factors rather than by contact-mediated mechanisms. Functionally, the intact CS tract has been linked with stride length of the hind limbs [2,20]. In our study we did not detect any recovery in stride length of the hind limbs in astrocyte transplanted animals versus controls. Obviously, an enhanced presence of injured CS axons rostral to a low-thoracic lesion gap is insufficient in stimulating recovery in this behavioral parameter.

In summary, our results demonstrate that CS axon regrowth into, across, and from a relatively large spinal lesion gap cannot be stimulated by a multifactorial transplantation strategy using aligned grafts of immature astrocytes on poly(D,L)-lactide matrices and astrocyte injections into the host spinal tissue. Future studies may focus on the nature of the die-back response in the presence of transplanted astrocytes. If the die-back response reflects a reduction in the number of axon retracting from the lesion gap, the transplantation strategy may strongly benefit from treatments enhancing survival of transplanted immature astrocytes within the spinal lesion gaps in its effect to stimulate long-distance axon regeneration across spinal lesion gaps.

\section{Acknowledgements}

The authors thank Marijke Lemmens for her assistance with the immunohistochemical stainings. The authors are also grateful to Lou Kirkels (IDEE, Maastricht) for his expertise with respect to the injection procedures. This work was funded by the International Spinal Research Trust (ISRT; STR057 to E.A.J.J.).

\section{References}

[1] D.M. Basso, M.S. Beattie, J.C. Bresnahan, A sensitive and reliable locomotor rating scale for open field testing in rats, J. Neurotrauma 2 (1) (1995) $1-21$.

[2] B.S. Bregman, E. Kunkel-Bagden, L. Schnell, H.N. Dai, D. Gao, M.E. Schwab, Recovery from spinal cord injury mediated by antibodies to neurite growth inhibitors, Nature 378 (6556) (1995) 498-501.

[3] M.I. Chuah, D. Choi-Lundberg, S. Weston, A.J. Vincent, R.S. Chung, J.C. Vickers, A.K. West, Olfactory ensheathing cells promote collateral axonal branching in the injured adult rat spinal cord, Exp. Neurol. 185 (1) (2004) 15-25. 
Published in: Neuroscience Letters (2006), vol. 400, iss. 3, pp. 208-212

Status : Postprint (Author's version)

[4] R. Deumens, G.C. Koopmans, C.G. Den Bakker, V. Maquet, S. Blacher, W.M. Hoing, R. Jérôme, J.R Pirard, H.W. Steinbusch, E.A. Joosten, Alignment of glial cells stimulates directional neurite growth of CNS neurons in vitro, Neuroscience 125 (3) (2004) $591-604$.

[5] R. Deumens, G.C. Koopmans, E.A. Joosten, Regeneration of descending axon tracts after spinal cord injury, Prog. Neurobiol. 77 (1-2) (2005) 57-89.

[6] S. Dijkstra, PR. Bar, W.H. Gispen, E.A. Joosten, Selective stimulation of dendrite outgrowth from identified corticospinal neurons by homotopic astrocytes, Neuroscience 92 (4) (1999) 1331-1342.

[7] Y. Goldshmit, M.P. Galea, G. Wise, P.F. Bartlett, A.M. Turnley, Axonal regeneration and lack of astrocytic gliosis in EphA4-deficient mice, J. Neurosci. 24 (45) (2004) 10064-10073.

[8] R. Grill, A. Blesch, F.H. Gage, M.H. Tuszynski, Cellular delivery of neurotrophin-3 promotes corticospinal axonal growth and partial functional recovery after spinal cord injury, J. Neurosci. 17 (1997) 5560-5572.

[9] F.P. Hamers, A.J. Lankhorst, T.J. van Laar, W.B. Veldhuis, W.H. Gispen, Automated quantitative gait analysis during overground locomotion in the rat: its application to spinal cord contusion and transection injuries, J. Neurotrauma 18 (2) (2001) 187-201.

[10] E.A. Joosten, AA. Gribnau, Astrocytes and guidance of outgrowing corticospinal tract axons in the rat. An immunocytochemical study using anti-vimentin and anti-glial fibrillary acidic protein, Neuroscience 31 (2) (1989) 439-452.

[11] E.A. Joosten, W.B. Veldhuis, F.P. Hamers, Collagen containing neonatal astrocytes stimulates regrowth of injured fibers and promotes modest locomotor recovery after spinal cord injury, J. Neurosci. Res. 77 (1) (2004) 127-142.

[12] G.C. Koopmans, R. Deumens, W.M. Honig, F.P. Hamers, H.W. Steinbusch, E.A. Joosten, The assessment of locomotor function in spinal cord injured rats: the importance of objective analysis of coordination, J. Neurotrauma 22 (2) (2005) 214-225.

[13] Y. Li, P.M. Field, G. Raisman, Repair of adult rat corticospinal tract by transplants of olfactory ensheathing cells, Science 277 (5334) (1997) 2000-2002.

[14] Y. Li, P.M. Field, G. Raisman, Regeneration of adult rat corticospinal axons induced by transplanted olfactory ensheathing cells, J. Neurosci. 18 (24)(1998) 10514-10524.

[15] P. Mainil-Varlet, R. Curtis, S. Gogolewski, Effect of in vivo and in vitro degradation on molecular and mechanical properties of various low-molecular-weight polylactides, J. Biomed. Mater. Res. 36 (3) (1997) 360-380.

[16] V. Maquet, D. Martin, F. Scholtes, R. Franzen, J. Schoenen, G. Moonen, R. Jérôme, Poly(D,L-lactide) foams modified by poly(ethylene oxide)-block-poly(D,L-lactide) copolymers and a-FGF: in vitro and in vivo evaluation for spinal cord regeneration, Biomaterials 22 (10) (2001) 1137-1146.

[17] P.G. Popovich, P. Wei, B.T. Stokes, Cellular inflammatory response after spinal cord injury in Sprague-Dawley and Lewis rats, J. Comp. Neurol. 377 (3) (1997) 443-464.

[18] A. Ramon-Cueto, M.I. Cordero, F.F. Santos-Benito, J. Avila, Functional recovery of paraplegic rats and motor axon regeneration in their spinal cords by olfactory ensheathing glia, Neuron 25 (2) (2000) 425-435.

[19] J.H. Skene, I. Virag, Posttranslational membrane attachment and dynamic fatty acylation of a neuronal growth cone protein, GAP-43, J. Cell Biol. 108 (2) (1989) 613-624.

[20] M.L. Starkey, A.W. Barritt, P.K. Yip, M. Davies, F.P. Hamers, S.B. McMahon, E.J. Bradbury, Assessing behavioural function following a pyramidotomy lesion of the corticospinal tract in adult mice, Exp. Neurol. 95 (2) (2005) 524-539.

[21] S.M. Strittmatter, T. Vartanian, M.C. Fishman, GAP-43 as a plasticity protein in neuronal form and repair, J. Neurobiol. 23 (5) (1992) 507-520.

[22] A. Woodhouse, A.J. Vincent, MA. Kozel, R.S. Chung, P.M. Waite, C. Vickers, A.K. West, M.I. Chuah, Spinal cord tissue affects ensheathing cell proliferation and apoptosis, Neuroreport 16 (7) (2005) 737-740. 